\title{
Differential turnover of the multiple processed transcripts of the Escherichia coli focA-pflB operon
}

\author{
Correspondence \\ R. Gary Sawers \\ sawers@staff.uni-marburg.de
}

Received 28 February 2006

Revised 9 April 2006

Accepted 11 April 2006
R. Gary Sawers $†$

Department of Molecular Microbiology, John Innes Centre, Norwich, UK

Expression of the anaerobically inducible focA-pflB operon of Escherichia coli is subject to complex transcriptional and post-transcriptional control, which generates eight transcripts whose $5^{\prime}$ ends span $\sim 1 \cdot 2 \mathrm{~kb}$. All eight transcripts have the same $3^{\prime}$ end. The $5^{\prime}$ ends of three of the transcripts, termed $6,6 \mathrm{a}$ and 7 , are located upstream of the operon. The promoters generating transcripts 6 and 7 are anaerobically regulated by FNR and ArcA $\sim P$, while promoter $6 a$ is constitutively active. The $5^{\prime}$ ends of the other five transcripts are all located within the operon. Most of the $5^{\prime}$ ends of these operon-internal transcripts result from RNA polymerase-dependent processing of the three longer primary transcripts, 6, 6a and 7. Here, it is demonstrated that subsequent to, and distinct from, these processing events, post-transcriptional modification of these transcripts also occurs through the action of the endoribonuclease RNase E. Transcripts 6 and 7 exhibit differential stability with half-lives of 1 and $5 \mathrm{~min}$, respectively. Transcript 7, which has the longer half-life, is the longest transcript of the operon and has a $\sim 340$ base untranslated leader. Two of the operon-internal transcripts, 4 and 5 , also have comparatively short half-lives in the wild-type, which are significantly increased in a mutant with impaired RNase E activity. A precursor-product relationship is observed between the longer transcripts 3-7 and transcripts 1 and 2. The $5^{\prime}$ ends of transcripts 1 and 2 are closest to the pflB gene and have half-lives of approximately 7-8 min. The consequence of this regulation is an accumulation of full-length pflB transcript and comparably low levels of dicistronic transcript. This ensures different levels of synthesis of the formate transporter FocA and pyruvate formate-lyase during anaerobic growth, while maintaining coordinate regulation. Transcript analysis throughout the growth phase revealed that maximal anaerobic expression of the focA-pflB operon was restricted to exponentially growing cells. Expression of transcript 7 peaked in early to mid-exponential phase, while the levels of transcript 6 steadily accumulated toward the late-exponential phase of growth. Taken together, these findings indicate that although subject to common positive control by ArcA $\sim \mathrm{P}$ and FNR, the transcripts generated by promoters 6 and 7 are subject to differential temporal and post-transcriptional regulation.

\section{INTRODUCTION}

In prokaryotes there are a number of levels at which gene expression is regulated. Transcriptional control is the primary means of regulation; however, post-transcriptional events such as RNA turnover can also exert regulatory control (Apirion \& Gegenheimer, 1984; Coburn \& Mackie, 1999). Since the mean half-life of mRNA species in bacteria like E. coli lies between 2 and $4 \mathrm{~min}$, control of mRNA turnover and degradation play key roles in influencing gene expression (Kennell, 2002; Kushner, 2002; Li \& Altman, 2004; Ow et al., 2002). In particular, differential

†Present address: Max-Planck-Institut für Terrestrische Mikrobiologie, Karl-von-Frisch-Straße, D-35043 Marburg, Germany

Abbreviations: PFL, pyruvate formate-lyase; URS, upstream regulatory sequence(s). endoribonucleolytic processing and stability of polycistronic transcripts allow modulation of the cellular concentrations of gene products while maintaining coordination of product synthesis (Coburn \& Mackie, 1999; Higgins \& Smith, 1986; Kennell, 2002; Kushner, 2002; Newbury et al., 1987). Usually these processing reactions form part of the general turnover cycle of mRNAs and regulation is a consequence of whether particular portions of transcripts are more or less susceptible to further degradation by exoribonucleases.

There are three major characterized cytoplasmic endoribonucleases in Escherichia coli, and these include the enzymes RNase III, RNase E and RNase G. While RNase III plays a major role in processing specific transcripts, its role in mRNA turnover is limited (Aristarkhov et al., 1996; Kushner, 2002). On the other hand, RNase E and its 
paralogue RNase $\mathrm{G}$ have major roles in mRNA turnover (Li et al., 1999; Tock et al., 2000; Umitsuki et al., 2001; Wachi et al., 1997) and both enzymes are conserved in a large number of prokaryotes (Kushner, 2002). The activity of RNases $\mathrm{E}$ and $\mathrm{G}$ is markedly affected by the presence of a $5^{\prime}$-end monophosphate on transcripts, and these transcripts are cleaved at a distant site (Bouvet \& Belasco, 1992; Coburn \& Mackie, 1999; Mackie, 1998; Tock et al., 2000). The recent structural determination of the catalytic domain of RNase E (Callaghan et al., 2005) has provided important insights into how the enzyme catalyses cleavage at a distance. RNase $\mathrm{E}$ is also associated through its C-terminal domain with a large protein complex called the 'RNA degradosome' (Carpousis, 2002); however, there is still considerable debate as to what role the 'degradosome' plays in mRNA turnover (Ow et al., 2000; Kennell, 2002; Kushner, 2002). RNase E initiates turnover of many mRNA species through selective processing at specific sites. The findings of this study show that RNase $\mathrm{E}$ is involved in initial events during the turnover of the anaerobically inducible $f o c A-p f l B$ transcript.

Expression of the focA-pflB operon is remarkably complex. The operon encodes the central enzyme of fermentative metabolism, pyruvate formate-lyase (PFL), and a membrane protein, FocA, thought to transport formate (Suppmann \& Sawers, 1994; Sawers \& Clark, 2004). Expression of the operon is subject to redox-dependent transcriptional activation by FNR and ArcA P (Sawers \& Suppmann, 1992; Sawers, 1993; Drapal \& Sawers, 1995; Kaiser \& Sawers, 1995). Transcription of the operon is also controlled from multiple promoters. Three of these promoters are located in the regulatory region $5^{\prime}$ of the operon, and two of these (promoters 6 and 7) are anaerobically regulated by FNR and ArcA $\sim \mathrm{P}$, while the third is a weakly transcribed constitutive promoter termed 6a (Fig. 1; Sawers \& Böck, 1989). The 5' ends of five further transcripts map within the operon. The

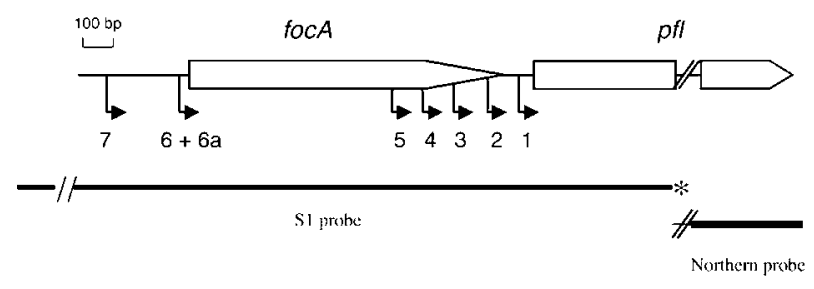

Fig. 1. Transcriptional organization of the foc $A-p f / B$ operon. $A$ schematic representation of the focA-pflB operon is shown along with an indication of the location of the $5^{\prime}$ ends of the transcripts, shown as angled arrows, generated. Transcripts 6, $6 a$ and 7 result from primary transcription events, while transcripts 1-5 mainly result from processing (see Sawers, $2005 \mathrm{~b}$ ). The locations of DNA probes used for S1 nuclease protection experiments and Northern blotting are shown. The probe for Northern blotting extended from the $\mathrm{BamHI}$ site at nucleotide position 390 within the pflB gene to position 2380 at the end of the gene. The scale bar is representative for the whole operon. $3^{\prime}$ ends of all eight transcripts map to the end of the pflB gene (Sawers \& Böck, 1989). Although the transcripts generated from promoters $6,6 \mathrm{a}$ and 7 are the result of primary transcription events (Sawers, 1993; Kaiser \& Sawers, 1997), recent studies have demonstrated that the majority of the five operon-internal transcripts result from specific processing of the longer primary transcripts (Sawers, 2005b). Surprisingly, these processing events appear to be RNApolymerase-dependent and although it is currently uncertain what factor(s) is responsible for this processing, it is clear that it is not RNase E, RNase G or RNase III. Nevertheless, it is possible that one or more of these endonucleases is involved in the general turnover of these transcripts subsequent to their generation. Since nothing is known regarding the stage in the growth phase that focA-pflB operon transcription occurs and what controls the initiation of transcript turnover and degradation, this study aimed to shed light on these events. As well as identifying a role for RNase $\mathrm{E}$ in transcript turnover, this work also shows that transcription of the operon is restricted to the exponential phase of growth, that the anaerobically inducible promoters 6 and 7 are expressed at different stages of the growth phase and that their transcripts have different stabilities.

\section{METHODS}

Bacterial strains, plasmids and culture conditions. Bacterial strains and plasmids used in this study are listed in Table 1. Bacteria were grown routinely at $37^{\circ} \mathrm{C}$ in the buffered rich medium TGYEP, pH 6.5, which included $20 \mathrm{mM}$ glucose (Begg et al., 1977). Where indicated, pyruvate was added to $10 \mathrm{mM}$ final concentration. Aerobic cultures were grown in flasks filled maximally to one-tenth of their volume, while anaerobic cultures were grown in stoppered bottles filled with medium to the top.

Cells for RNA analysis were generally grown to mid-exponential phase $\left(\mathrm{OD}_{600}=0 \cdot 4-0 \cdot 5\right)$ prior to harvest. In the case where RNA was isolated from late-stationary-phase cells, cultures were grown for $16 \mathrm{~h}$ prior to harvest.

Antibiotics were added to the following final concentrations $\left(\mu \mathrm{g} \mathrm{ml}^{-1}\right)$ : ampicillin, 75 ; chloramphenicol, 15; tetracycline, 15 . Media were solidified by the inclusion of $1 \cdot 5 \%(\mathrm{w} / \mathrm{v})$ agar.

Analysis of RNA transcripts. Two methods were used to isolate total RNA from cells. The standard method involved use of a Qiagen RNeasy kit, according to the manufacturer's instructions. In experiments where the half-life of the $f \circ c A-p f l B$ transcripts was analysed, cells were grown anaerobically to an $\mathrm{OD}_{600}$ of 0.5 and rifampicin was added to the cultures to a final concentration of $0.2 \mathrm{mg} \mathrm{ml}^{-1}$. To obtain the $0 \mathrm{~min}$ time-point, immediately prior to addition of rifampicin, a $5 \mathrm{ml}$ aliquot of the culture was removed and rapidly added to $5 \mathrm{ml}$ hot $\left(65^{\circ} \mathrm{C}\right)$ phenol (Aiba et al., 1981). At appropriate time-points after rifampicin addition, a $5 \mathrm{ml}$ aliquot of the culture was removed and immediately added to $5 \mathrm{ml}$ hot phenol for RNA preparation. In experiments where RNA was isolated from $\mathrm{CH} 1826$ ( $r n e-1)$, cells were grown to an $\mathrm{OD}_{600}$ of approximately 0.5 at $30{ }^{\circ} \mathrm{C}$ and then cultures were shifted to $42^{\circ} \mathrm{C}$ for $30 \mathrm{~min}$ prior to harvesting of cells for RNA preparation or addition of rifampicin to determine the chemical half-life of transcripts. The chemical half-life determinations were performed at least twice for each strain and representative gels are show for each experiment. 
Table 1. Strains and plasmids used in this study

\begin{tabular}{|c|c|c|}
\hline Strain/plasmid & Genotype $^{*}$ & Reference \\
\hline RM4100 & Like MC4100 but with wild-type $f n r$ gene regulatory region & Sawers $(2005 a)$ \\
\hline $\mathrm{CH} 1827$ & araD139 $\Delta($ araABC-leu $) 7697 \Delta($ lac $) X 74$ galU galK hsdR rpsL150 thi zce-726: :Tn10 & Mudd et al. (1990) \\
\hline RM6521 & Like MC4100 but rnc14::Tn10 & Sawers $(2005 b)$ \\
\hline p29 & $\mathrm{Cm}^{\mathrm{R}} f o c A^{+} p f l B^{+} a c t^{+}$ & Christiansen \& Pedersen (1981) \\
\hline pBR322 & $\mathrm{Ap}^{\mathrm{R}} \mathrm{Tc}^{\mathrm{R}}$ & Bolivar et al. (1977) \\
\hline
\end{tabular}

${ }^{\star}$ Ap, Ampicillin; Cm, chloramphenicol; Tc, tetracycline.

S1 nuclease mapping of focA-pflB transcripts was performed according to either Sawers \& Böck (1989) or Sawers (2005b) using $50 \mu$ g total RNA. The BamHI DNA fragment used for hybridization was derived from plasmid p29 (Christiansen \& Pedersen, 1981). Labelling of this fragment with $\left[\gamma-{ }^{32} \mathrm{P}\right] \mathrm{ATP}$, as well as treatment of the fragment prior to S1 analysis, was carried out exactly as described previously (Sawers, 2005b).

Northern blotting of total RNA was performed according to Sawers \& Böck (1989). A total of $10 \mu \mathrm{g}$ total RNA from each sample was applied to a $1 \%(\mathrm{w} / \mathrm{v})$ agarose gel containing formaldehyde. The $\sim 2 \mathrm{kbp}$ DNA probe used to detect the $p f l B$ transcripts is shown in Fig. 1 and was labelled by nick-translation (Sambrook et al., 1989) using $\left[\alpha-{ }^{32} \mathrm{P}\right] \mathrm{dATP}$.

Other methods. Protein concentration was determined by the Lowry method. SDS-PAGE was performed using 7.5\% (w/v) polyacrylamide gels according to the method of Laemmli (1970). Western blotting was carried out according to Towbin et al. (1979). Anti-PFL antiserum was diluted 1:10000 and the antibody-antigen reaction was visualized using the ECL chemiluminescent method (Amersham Bioscience), exactly as recommended by the manufacturer. These experiments were repeated at least twice and representative gels are shown. Densitometric analyses were performed using SynGene Gene Tools analysis software.

\section{RESULTS}

\section{High focA-pflB transcript levels in exponential- phase cells}

Multiple transcripts control expression of the focA-pflB operon (Fig. 1). Five of these transcripts result from processing of three longer transcripts, which originate in the upstream regulatory sequences (URS) (Fig. 1). These processing events are catalysed by a currently unknown factor(s), but they appear to be distinct from RNA turnover and degradation events (Sawers, 2005b). To determine precisely when during the growth phase the focA-pflB operon is expressed, total RNA from cells grown aerobically and anaerobically in the exponential and stationary phase was isolated and analysed by S1 nuclease protection (Fig. 2). Analysis of RNA from aerobic cells (Fig. 2, lane 1) grown to mid-exponential phase revealed low levels of transcripts $1-5$, with barely detectable levels of transcript 6A (anaerobically induced, FNR-dependent transcript 6 is not present in aerobically grown cells: Sawers, 1993; Reyes-Ramírez \& Sawers, 2006). Transcript 7 is never observed in aerobically grown cells (Sawers, 1993; Reyes-Ramírez \& Sawers, 2006). Supplementation of the growth medium with pyruvate, which was shown previously to activate $p f l B$-lacZ expression (Sawers \& Böck, 1989), had only a marginal effect in slightly increasing the levels of transcript 6A (Fig. 2, lane 2). RNA isolated from cells grown aerobically with glucose into late stationary phase had a slightly altered transcript pattern where the level of transcript 6 or $6 a$ (this gel system does not distinguish these transcripts) increased approximately threefold relative to the level in exponential-phase cells (Fig. 2, lane 3). This is probably due to increased transcription from promoter 6 , as no such increase in transcript level was observed when glucose was omitted from the growth medium (see lane 4) and transcript 6a has been shown to be constitutively transcribed (Kaiser \& Sawers, 1997).

Notably, the level of transcript 5 in aerobic stationary-phase cells grown on glucose also increased relative to the level in exponentially growing cells, while the levels of transcripts 1 and 2 were clearly reduced. This might suggest that the processing activity responsible for cleavage of the transcripts originating in the URS is maximal in cells growing in the exponential phase (see also below).

Anaerobic growth to exponential phase showed substantial induction of transcript levels (Fig. 2, lanes 5 and 6), which is consistent with previous observations (Sawers \& Böck, 1989). While the levels of all transcripts increased coordinately relative to levels during aerobic growth, transcript 7 was only detectable under anaerobic conditions. Pyruvate had no discernible effect on the overall transcript pattern (Fig. 2, lane 6).

In cells grown anaerobically to late stationary phase, transcripts 4, 5, 6 and 7 were not detectable and transcripts 1, 2 and 3 were dramatically reduced in level compared with exponentially grown cells. This finding indicates that focA$p f l B$ operon transcription is virtually non-existent in late stationary phase. This result contrasts with what was 
S 122344567

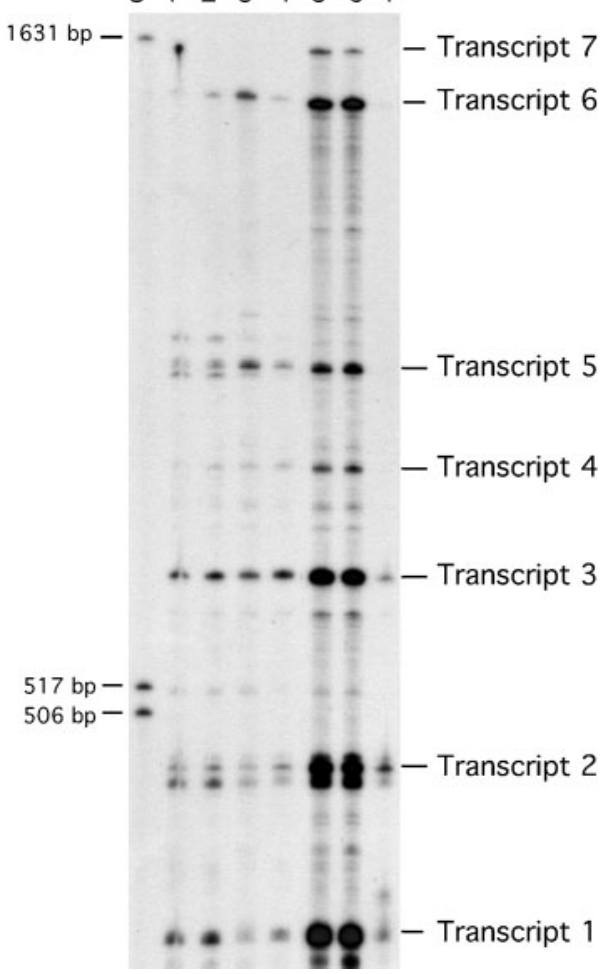

Fig. 2. $S 1$ nuclease protection analysis of $f \circ c A-p f l B$ transcripts generated after growth of wild-type $E$. coli to mid-exponential and late stationary phase in the presence and absence of oxygen. Total RNA was isolated from strains grown in TGYEP medium and hybridized with radioactively labelled DNA as described in Methods. Lanes: S, radioactively labelled DNA size standards generated by digestion of pBR322 with Hinfl; 1, S1 nuclease analysis of total RNA isolated from cells grown aerobically to mid-exponential phase $\left(O D_{600}=0 \cdot 4-0.5\right)$ of growth; 2 , as lane 1 but the growth medium was supplemented with $10 \mathrm{mM}$ pyruvate; 3 , as lane 1 but RNA was isolated from cells grown to late stationary phase ( $16 \mathrm{~h}$ growth); 4 , as lane 3 but without addition of glucose or pyruvate; 5, RNA isolated from cells grown anaerobically in TGYEP medium to mid-exponential phase of growth; 6 , as lane 5 but the growth medium was supplemented with $10 \mathrm{mM}$ pyruvate; 7, as lane 5 but RNA was isolated from cells grown to late stationary phase $(16 \mathrm{~h}$ growth). The location of the $5^{\prime}$ ends of the transcripts is indicated on the right. Samples were separated in a denaturing $4 \%(\mathrm{w} / \mathrm{v})$ polyacrylamide gel. Note that the transcripts generated from promoters 6 and $6 \mathrm{~A}$ are not separated in this gel system and are thus collectively referred to as transcript 6 .

observed in aerobically grown cells and suggests that transcription initiation was reduced under these conditions in anaerobic cells and/or that the transcripts were degraded.

To determine more precisely in the growth phase when the focA-pflB transcript levels started to decrease during anaerobiosis, RNA was prepared from cells throughout growth, from early exponential to early stationary phase (Fig. 3). S1 nuclease protection analysis revealed that even in cells that were emerging from the lag phase (Fig. $3 \mathrm{~b}$, lane 1), anaerobic focA-pflB transcription was activated. Moreover, the pattern of transcripts 1-6 was similar until late-exponential phase (Fig. 3b, lane 5), indicating that there was no difference in the level of co-transcriptional processing. Interestingly, expression of transcript 7 peaked at early to mid-exponential phase (Fig. 3b, lanes 2 and 3). On the other hand, the intensity of transcript 6 , as determined by densitometric analysis, peaked at late exponential phase (Fig. 3b, lane 5). As soon as cells entered stationary phase, transcript 7 was no longer detectable and the levels of transcripts 4, 5 and 6 were substantially reduced (Fig. 3b, lane 6). This suggested either that transcription of the operon was reduced or that turnover of the transcripts was significantly increased, or that both of these events contributed to reduced levels of the transcripts.

Since the S1 nuclease protection analyses undertaken here only provide information about the $5^{\prime}$ ends of transcripts, it was important to examine the levels of full-length $p f l B$ transcripts during the growth phase. The same RNA samples were analysed in a Northern blot experiment (Fig. 3c). The level of the full-length $p f l B$ transcript was clearly reduced in stationary phase in accord with what was observed in the S1 nuclease protection experiment.

A Western blot using anti-PFL antiserum revealed that the level of PFL polypeptide was approximately fivefold lower in the lag phase (Fig. 3d, lane 1) compared with the maximal level observed in late exponential phase (Fig. 3d, lane 5). The level of PFL polypeptide began to decrease in early stationary phase, but was still present at significant levels, suggesting that the half-life of the protein is long. Notably, the Western blot revealed a protein doublet, the lower band of which, termed $\mathrm{PFL}^{\prime}$, is characteristic of radical-bearing enzyme that has undergone oxygenolytic cleavage (Knappe \& Sawers, 1990; Wagner et al., 1992) and indicates that the polypeptide in early stationary phase was biologically active.

\section{Differential half-lives of focA-pflB transcript $5^{\prime}$ ends}

The half-lives of the $5^{\prime}$ ends of seven of the eight focA-pflB transcripts were determined using rifampicin to inhibit DNA-dependent RNA polymerase transcription initiation (see Methods) and S1 nuclease protection to analyse the complete set of transcripts (Fig. 4). RNA was analysed from cultures grown to the mid-exponential phase, equivalent to an $\mathrm{OD}_{600}$ of $\sim 0.5$ (see sample 2 in Fig. 3a). The transcripts exhibited different turnover profiles in response to rifampicin treatment. Whilst transcript 6 was rapidly degraded with a half-life of $1 \mathrm{~min}$ (see Fig. 4a, b), the half-life of transcript 7 was shown reproducibly to be between 5 and $6 \mathrm{~min}$. This finding further emphasizes the different regulation of these transcripts. Transcript 5 also had a similar turnover profile to that of transcript 6 and thus had a similar half-life of approximately $1 \mathrm{~min}$. The remaining transcripts exhibited a precursor-product relationship, in particular transcript 1 , the level of which increased during the course of 
(a)

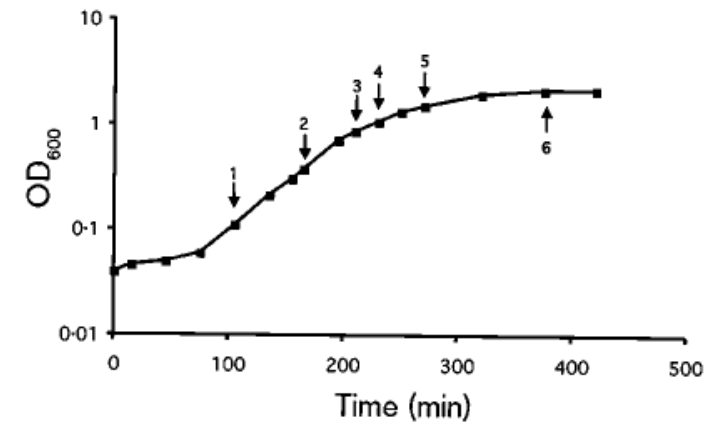

(b)

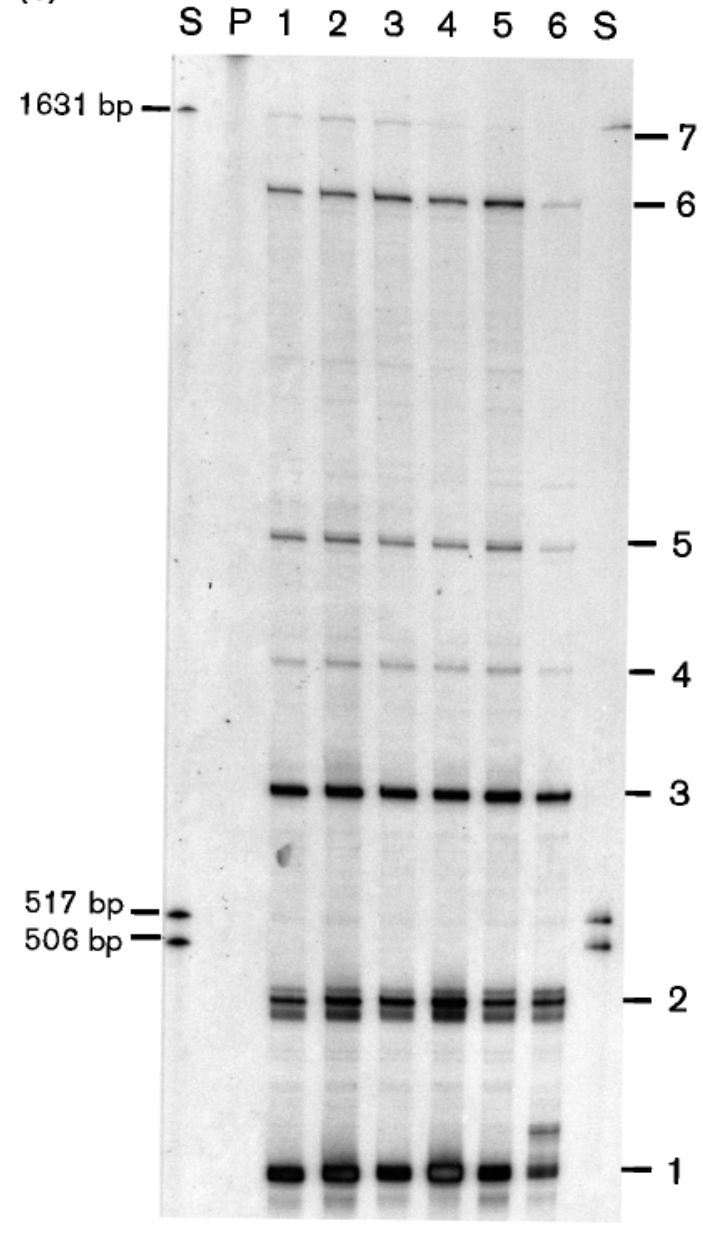

(c) $\begin{array}{llllll}1 & 2 & 3 & 4 & 5 & 6\end{array}$

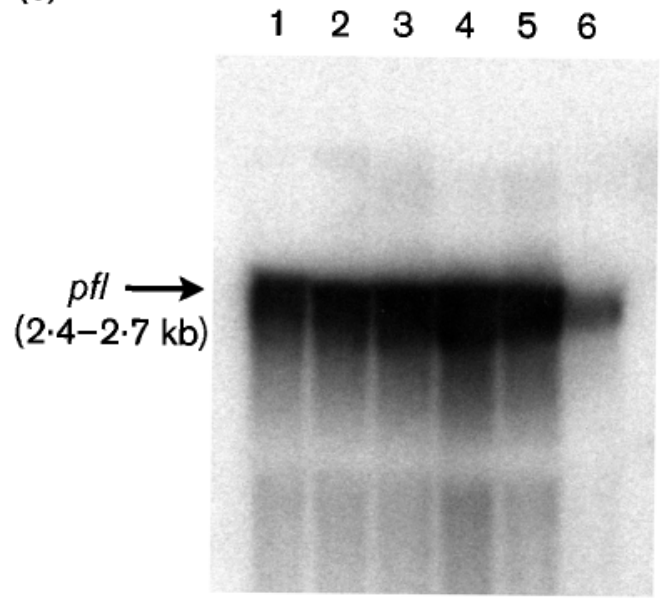

rRNA

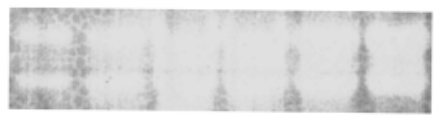

(d)

Fig. 3. The focA-pflB transcript levels are maximal in exponentially growing anaerobic cells of $E$. coli. (a) Graphical representation of anaerobic growth of $E$. coli in TGYEP medium. The vertical arrows indicate times when samples of cells were removed from the culture and total RNA isolated for $\mathrm{S} 1$ nuclease analysis. (b) $\mathrm{S} 1$ nuclease analysis of focA-pflB transcripts generated throughout the anaerobic growth phase. Lanes: $S$, DNA size markers (see legend to Fig. 2); $P$, radioactively labelled DNA probe used for the S1 analysis; 1-6, RNA samples isolated from cells harvested at points 1-6, respectively, in (a). The numbers 1-7 on the right indicate the locations of transcripts 1-7, respectively. (c). Northern analysis of full-length pfIB transcripts. Total RNA from samples of the culture shown in (a) were separated in a denaturing agarose gel, transferred to nylon membrane and hybridized with radioactively labelled DNA from the pfIB gene as described in Methods (see Fig. 1). Lanes: 1-6, RNA samples isolated from the culture at positions 1-6 in (a). The location and size range of the full-length pflB transcript are indicated. An image of the $16 \mathrm{~S}$ and $23 \mathrm{~S}$ rRNA species from the same gel and visualized by staining with acridine orange is shown below the Northern blot. The order of the lanes is identical to that in the upper panel. The weak bands above the main pflB signals represent 3.2-3.6 kb focA-pflB transcripts. (d) A western blot of the six culture samples used for RNA preparation and for visualizing the PFL antigen is shown. Whole-cell lysates (100 $\mu \mathrm{g}$ protein) were subjected to $7 \cdot 5 \%(\mathrm{w} / \mathrm{v})$ SDS-PAGE. PFL and PFL' represent full-length and oxygenolytically cleaved species of the protein, respectively. For details of the Western blotting procedure, see Methods. 
(a)

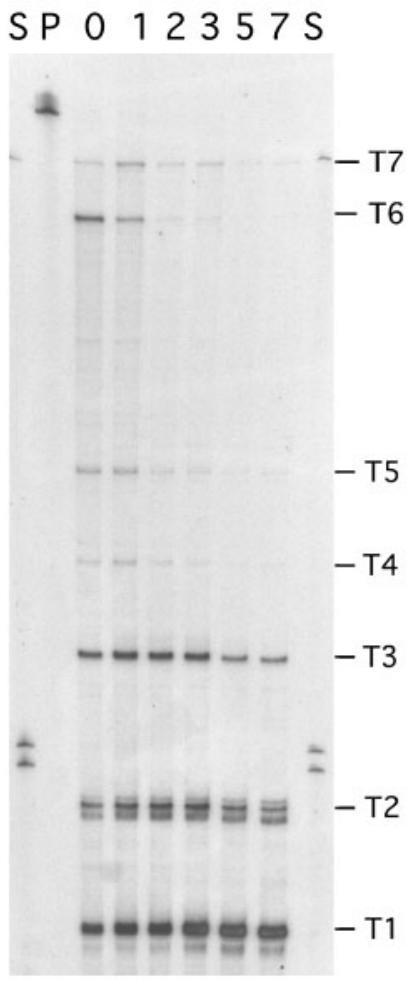

(b)

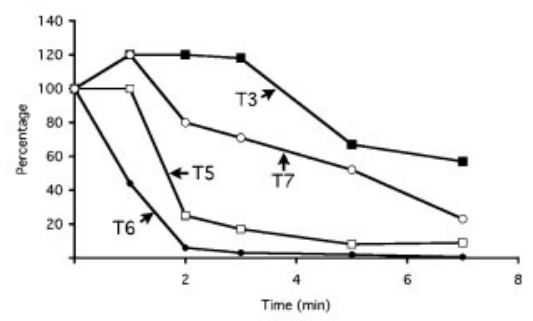

(c)

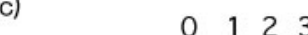

rRNA
Fig. 4. Determination of the half-life of the $5^{\prime}$ ends of the focA-pflB transcripts. (a) $\mathrm{S} 1$ nuclease analysis of $f \circ c A-p f / B$ transcripts after the addition of rifampicin to inhibit further transcription initiation. Lanes: $\mathrm{S}$, radioactive DNA size markers (see legend to Fig. 2); $\mathrm{P}$, radioactively labelled DNA probe used in the $S 1$ nuclease analyses; 0-7, labelled to indicate the time ( $\mathrm{min}$ ) after addition of rifampicin. (b) The results of densitometric analysis of selected transcripts are shown graphically. The intensity level of each transcript at time point 0 min was taken as $100 \%$ and the intensity of each transcript at subsequent time points was determined. In the interests of clarity, the data for only transcripts $3,5,6$ and 7 are shown and are labelled T3, T5, T6 and T7, respectively. (c) Northern analysis of full-length pflB transcripts. Total RNA from samples of the culture shown in (a) was separated in a denaturing agarose gel, transferred to a nylon membrane and hybridized with radioactively labelled DNA from the pfIB gene as described in Methods. Lanes are labelled with respect to the time points of RNA sample isolation from the culture at $0,1,2,3,5$ and $7 \mathrm{~min}$ after rifampicin addition. The location and size range of the full-length $p f l B$ transcript are indicated. An image of the $16 \mathrm{~S}$ and $23 \mathrm{~S}$ rRNA species from the same gel and visualized by staining with acridine orange is shown below the Northern blot. The order of the lanes is identical to that in the upper panel. The weak bands above the main $p f l B$ signals represent $3 \cdot 2-3 \cdot 6 \mathrm{~kb}$ focA-pflB transcripts. the experiment (Fig. 4a). These findings indicated that transcript 1 was a degradation-intermediate for at least some of the longer transcripts.

The accumulation of transcript 1 suggested that the half-life of the full-length $p f l B$ transcript was considerably longer than that of the full-length focA-pflB transcript. A Northern blot analysis of the $p f l B$ transcript revealed that the half-life was approximately 7-8 $\mathrm{min}$, which is in agreement with the S1 nuclease protection analysis of transcripts 1,2 and 3 (Fig. 4c).

\section{RNase $E$ is responsible for focA-pflB transcript turnover}

While the RNases III, E and G have been shown not to be involved in the initial co-transcriptional processing of the focA-pflB transcripts (Sawers, 2005b), it was important to determine whether any of them had a role in turnover of the transcripts. The half-lives of the $5^{\prime}$ ends of the transcripts were therefore analysed in various RNase mutant backgrounds (Fig. 5a). Transcripts 4, 5 and 6 were no longer detectable in the wild-type, and the rng and $r n c$ mutants, after $10 \mathrm{~min}$ treatment with rifampicin. Indeed, the transcript profiles were essentially identical (Fig. 5a). In contrast, in a mutant with a temperature-sensitive RNase E enzyme, transcripts 4,5 and 6 could readily be detected after $10 \mathrm{~min}$ treatment with rifampicin. Densitometric analysis of the transcripts indicated that the half-lives of transcripts 5 and 6 had increased from $1 \mathrm{~min}$ in the wild-type to approximately $5 \mathrm{~min}$ in the rne-1 mutant (data not shown). Moreover, transcripts 1 and 2 had neither diminished in level nor accumulated intermediates on the degradation pathway. This finding indicates that RNase $\mathrm{E}$ has an important role minimally in the turnover of the $5^{\prime}$ ends of the focA-pflB transcripts.

\section{DISCUSSION}

This study has highlighted several new features of $f o c A-p f l B$ operon regulation. First, operon transcription under 


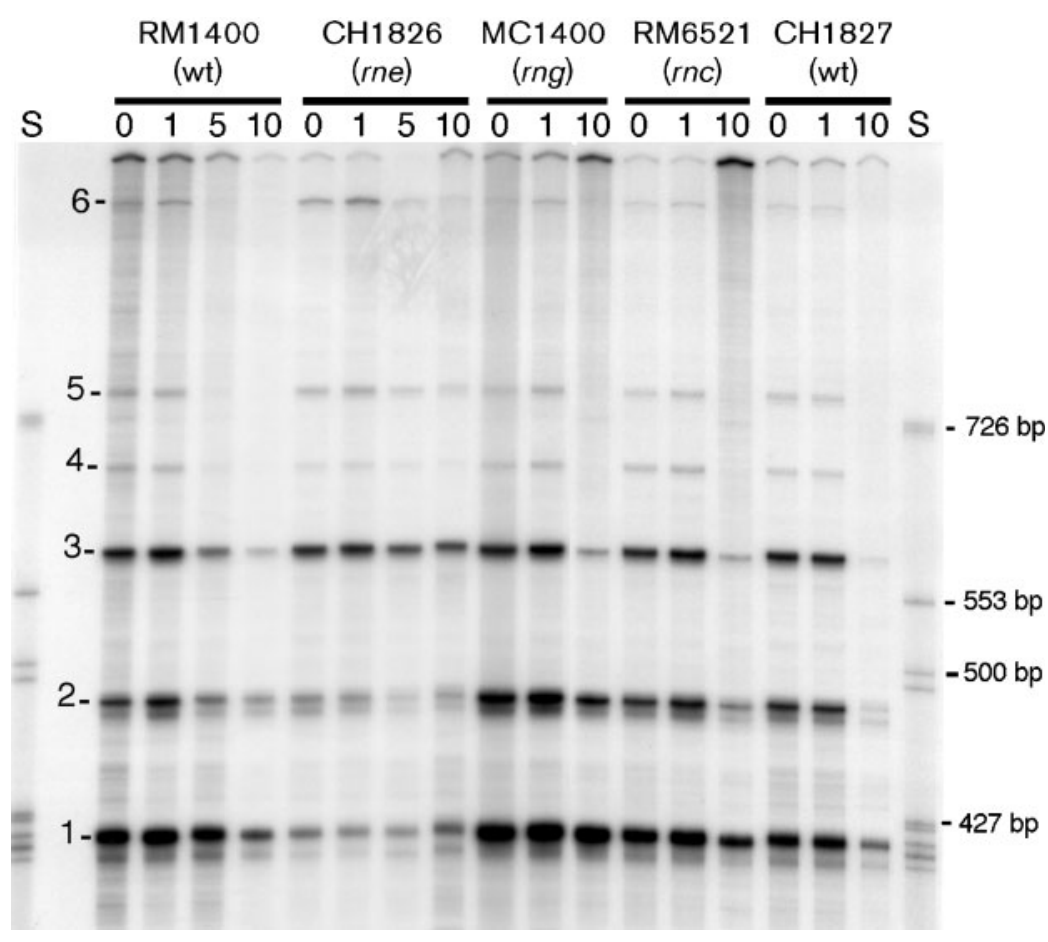

Fig. 5. $S 1$ nuclease analysis of foc $A-p f / B$ transcript stability in mutants affected in RNA turnover. (a) Total RNA was isolated from $E$. coli strains treated for different lengths of time with rifampicin and analysed by $\mathrm{S} 1$ nuclease protection as described in Methods. The times (min) after rifampicin treatment prior to RNA isolation are shown above each lane and the strains in which the experiments were conducted are indicated. The horizontal bar delimits the experimental series for a particular strain. The locations of the individual transcripts in the gel are indicated on the left of the figure as $1-6$. Note that in this series of experiments, S1 nuclease treatment was done by a method that does not clearly identify transcript 7. The signal at the top of the gel represents undigested radioactive DNA probe. Lane S, DNA size markers generated by digesting $\Phi \times 174$ with Hinfl. The sizes of the respective fragments are shown on the right of the figure.

anaerobic growth conditions is restricted to exponentially growing cells; second, transcripts with different $5^{\prime}$ ends are subject to differential turnover and degradation; third, one of the enzymes that is involved in this turnover is RNase E; fourth, the transcript derived from promoter 6 is turned over rapidly, while the longer transcript derived from promoter 7 is degraded five times more slowly; and finally, the combined processing and turnover of the multiple transcripts has the consequence that although FocA and PFL synthesis remains coordinated, comparatively higher amounts of PFL polypeptide can be produced.

One of the unusual features of the focA-pflB operon is the long URS, which comprises minimally $416 \mathrm{bp}$ with an untranslated region of 346 bases between the $5^{\prime}$ end of transcript 7 and the GUG translation initiation codon of the focA gene (Sawers \& Böck, 1989). Within this URS are three promoters, the weak, constitutive P6a promoter (Kaiser \& Sawers, 1997), and the anaerobically inducible P6 and P7 promoters (Sawers, 1993; Drapal \& Sawers, 1995). Both P6 and P7 are regulated by FNR and ArcA $\sim \mathrm{P}$, but while the upstream $\mathrm{P} 7$ promoter is absolutely dependent on both FNR and $\operatorname{ArcA} \sim \mathrm{P}$, the strong $\mathrm{P} 6$ promoter is still functional in the absence of ArcA $\sim \mathrm{P}$. Until this study, this was the only regulatory distinction between these promoters. The transcription start site of the $\mathrm{P} 7$ promoter is far upstream of the focA gene and the function of this promoter in governing operon expression has been an enigma, mainly because of its location, but also because the activity of the promoter appears to be constrained (Drapal \& Sawers, 1995). Here it is clearly shown that the level of the P7 transcript peaks in the early exponential phase of growth, with the level becoming reduced in the mid- to late exponential phase. In contrast, the level of P6-generated transcript slowly increased until the late exponential phase of growth, where it peaked, and then decreased rapidly upon entry into stationary phase. Based on this experiment alone, it is not possible to state unequivocally that the temporal difference in P6 and P7 transcript levels is a consequence of a difference in transcription activation, since the absolute transcript level observed is the sum of transcription plus turnover. The results of the transcription inhibition experiment, however, revealed that transcript 7 has a comparatively long half-life of approximately $5 \mathrm{~min}$, while that of transcript 6 was only of the order of $1 \mathrm{~min}$. Whilst it cannot be excluded that specific transcript stability may change throughout the growth phase, this finding suggests that the differences in temporal regulation observed for transcripts 6 and 7 are through transcriptional control, indicating that these promoters are responding in their activity to different signals. Since both are regulated positively by ArcA $\sim \mathrm{P}$ and FNR, the difference in their regulation could be due to either promoter 7 functioning only when both ArcA $\sim \mathrm{P}$ and FNR are maximally active, or a further factor to which this particular promoter responds.

It is intriguing that transcript 7 , which has a 346 base untranslated region, has a significantly longer half-life than transcript 6, which lies downstream of it and is within 26 bases of the focA gene. This difference in stability might be conferred by the additional 320 bases of AU-rich sequence at the $5^{\prime}$ end of the P7 transcript.

The rapid degradation of transcripts 4 and 5 is also slowed markedly by the temperature-sensitive rne-1 mutation, suggesting that they are also substrates for RNase E. At least in 
the case of transcripts 1 and 2 it appears clear that they are likely intermediates in the degradation of the longer focA$p f l B$ transcripts (Fig. 6) and they act as a barrier to further rapid degradation of the full-length $p f l B$ transcript. The factor(s) determining the comparative longevity of these transcripts is also currently unclear. Northern and Western blotting experiments indicated that the $p f l B$ transcripts and the PFL protein are relatively long-lived species in the growing anaerobic cell. This correlates well with the key role that PFL plays in primary metabolism during both fermentative and anaerobic respiratory growth (Sawers \& Clark, 2004). The formate channel FocA is required in lower amounts and presumably the amount synthesized from the weakly expressed but long-lived dicistronic focA-pflB transcript is sufficient to meet the requirements of the cell to export and reimport formate (Suppmann \& Sawers, 1994). Further work will be required to determine the precise level of FocA in the cell.

It is important to emphasize that $\mathrm{RNase} \mathrm{E}$ is not responsible for the initial processing of the focA-pflB transcript, which is a co-transcriptional event and appears to be somehow associated with RNA polymerase (Sawers, 2005b), since in an rne-1 mutant grown at the non-permissive temperature,

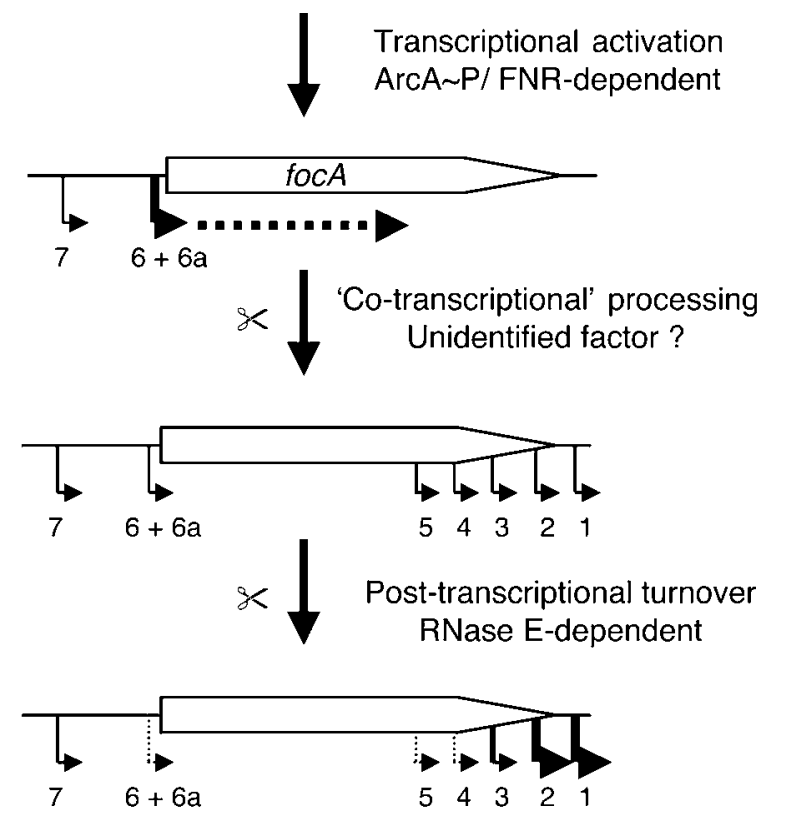

Fig. 6. Schematic representation of the events underlying the transcriptional control of the focA-pflB operon. Only the $5^{\prime}$ end of the operon is shown. The angled arrows represent the locations of the $5^{\prime}$ ends of the transcripts and the thickness of the arrow gives an indication of transcript intensity. The dotted horizontal arrow in the top panel indicates transcriptional orientation and the fact that transcription from the upstream regulatory sequence is required for the appearance of the downstream, processed transcripts 1-5. The dotted, angled arrows indicate transcript ends particularly susceptible to degradation by RNase E. the characteristic pattern of eight transcripts (including transcript 6a) is observed. Nevertheless, it appears that RNase E contributes to the production of the comparatively stable transcripts 1 and 2, since their abundance in the rne-1 mutant was significantly reduced compared with the wildtype. It will be of interest to determine whether it functions alone or as part of the 'degradosome' in its capacity to turnover the focA-pflB transcript.

\section{ACKNOWLEDGEMENTS}

Dr Roger Harris is thanked for his help with the densitometric analyses. This work was supported by a competitive strategic grant to the John Innes Centre from the Biotechnology and Biological Sciences Research Council.

\section{REFERENCES}

Aiba, H., Adhya, S. \& de Crombrugghe, B. (1981). Evidence for two functional gal promoters in intact Escherichia coli cells. J Biol Chem 256, 11905-11910.

Apirion, D. \& Gegenheimer, P. (1984). Molecular biology of RNA processing in prokaryotic cells. In Processing of RNA, pp. 36-52. Edited by D. Apirion. Boca Raton, FL: CRC Press.

Aristarkhov, A., Mikulskis, A., Belasco, J. G. \& Lin, E. C. C. (1996). Translation of the adhE transcript to produce ethanol dehydrogenase requires RNaseIII cleavage in Escherichia coli. J Bacteriol 178, 4327-4332.

Begg, Y. A., Whyte, J. N. \& Haddock, B. A. (1977). The identification of mutants of Escherichia coli deficient in formate dehydrogenase and nitrate reductase activities using dye indicator plates. FEMS Microbiol Lett 2, 47-50.

Bolivar, F., Rodriguez, R. L., Greene, P. J., Betlach, M. C., Heynecker, H. L., Boyer, H. W., Crosa, J. H. \& Falkow, S. (1977). Cloning and characterisation of new cloning vehicles. II A multipurpose system. Gene 2, 95-113.

Bouvet, P. \& Belasco, J. G. (1992). Control of RNase E-mediated RNA degradation by $5^{\prime}$-terminal base pairing in E. coli. Nature 360, 488-492.

Callaghan, A. J., Marcaida, M. J., Stead, J. A., McDowall, K. J., Scott, W. G. \& Luisi, B. F. (2005). Structure of Escherichia coli RNase E catalytic domain and implications for RNA turnover. Nature 437, 1187-1191.

Carpousis, A. J. (2002). The Escherichia coli RNA degradosome: structure, function and relationship in other ribonucleolytic multienzyme complexes. Biochem Soc Trans 30, 150-155.

Casadaban, M. J. \& Cohen, S. N. (1979). Lactose genes fused to exogenous promoters in one step using Mu-lac bacteriophage: in vivo probe for transcriptional control sequences. Proc Natl Acad Sci U S A 76, 4530-4533.

Christiansen, L. \& Pedersen, S. (1981). Cloning, restriction endonuclease mapping and post-transcriptional regulation of $\operatorname{rps} A$, the structural gene for ribosomal protein S1. Mol Gen Genet 181, 548-551.

Coburn, G. A. \& Mackie, G. A. (1999). Degradation of mRNA in Escherichia coli: an old problem with some new twists. Prog Nucleic Acid Res Mol Biol 62, 55-108.

Drapal, N. \& Sawers, G. (1995). Promoter 7 of the Escherichia coli pfl operon is a major determinant in the anaerobic regulation of expression by ArcA. J Bacteriol 177, 5338-5341. 
Higgins, C. F. \& Smith, N. H. (1986). Messenger RNA processing, degradation and the control of gene expression. In Regulation of Gene Expression - 25 Years On (Society for General Microbiology Symposium no. 39), pp. 179-198. Edited by I. R. Booth \& C. F. Higgins. Cambridge: Cambridge University Press.

Kaiser, M. \& Sawers, G. (1995). FNR activates transcription from the P6 promoter of the pfl operon in vitro. Mol Microbiol 18, 331-342.

Kaiser, M. \& Sawers, G. (1997). Overlapping promoters modulate Fnr- and ArcA-dependent anaerobic transcriptional activation of the focApfl operon in Escherichia coli. Microbiology 143, 775-783.

Kennell, D. (2002). Processing endoribonucleases and mRNA degradation in bacteria. J Bacteriol 184, 4645-4657.

Knappe, J. \& Sawers, G. (1990). A radical route to acetyl-CoA: the anaerobically induced pyruvate formate-lyase system of Escherichia coli. FEMS Microbiol Rev 75, 383-398.

Kushner, S. R. (2002). mRNA decay in Escherichia coli comes of age. J Bacteriol 184, 4658-4665.

Laemmli, U. K. (1970). Cleavage of structural proteins during assembly of the head of bacteriophage T4. Nature 227, 680-685.

Li, Y. \& Altman, S. (2004). Polarity effects in the lactose operon of Escherichia coli. J Mol Biol 339, 31-39.

Li, Z., Pandit, S. \& Deutscher, M. P. (1999). RNase G (CafA protein) and RNase E are both required for the $5^{\prime}$ maturation of $16 \mathrm{~S}$ ribosomal RNA. EMBO J 18, 2878-2885.

Mackie, G. A. (1998). Ribonuclease $\mathrm{E}$ is a $5^{\prime}$-end-dependent endonuclease. Nature 395, 720-723.

Mudd, E. A., Krisch, H. M. \& Higgins, C. F. (1990). RNase E, an endoribonuclease, has a general role in the chemical decay of Escherichia coli mRNA: evidence that rne and ams are the same genetic locus. Mol Microbiol 4, 2127-2135.

Newbury, S. F., Smith, N. H. \& Higgins, C. F. (1987). Differential mRNA stability controls relative gene expression within a polycistronic operon. Cell 51, 1131-1143.

Ow, M. C., Liu, Q. \& Kushner, S. R. (2000). Analysis of mRNA decay and rRNA processing in Escherichia coli in the absence of RNase E-based degradosome assembly. Mol Microbiol 38, 854-866.

Ow, M. C., Liu, Q., Mohanty, B. K., Andrew, M. E., Maples, V. F. \& Kushner, S. R. (2002). RNase E levels in Escherichia coli are controlled by a complex regulatory system that involves transcription of the rne gene from three promoters. Mol Microbiol 43, 159-171.

Reyes-Ramírez, F. \& Sawers, R. G. (2006). Aerobic activation of the anaerobically inducible Escherichia coli focA-pfl operon transcription by FNR. FEMS Microbiol Lett 255, 262-267.
Sambrook, J., Fritsch, E. F. \& Maniatis, T. (1989). Molecular Cloning: a Laboratory Manual. Cold Spring Harbor, NY: Cold Spring Harbor Laboratory.

Sawers, G. (1993). Specific transcriptional requirements for positive regulation of the anaerobically inducible $p f l$ operon by ArcA and FNR. Mol Microbiol 10, 737-747.

Sawers, R. G. (2005a). Expression of $f n r$ is constrained by an upstream IS5 insertion in certain Escherichia coli K-12 strains. J Bacteriol 187, 2609-2617.

Sawers, R. G. (2005b). Evidence for novel processing of the anaerobically inducible dicistronic focA-pfl mRNA transcript in Escherichia coli. Mol Microbiol 58, 1441-1453.

Sawers, G. \& Böck, A. (1989). Novel transcriptional control of the pyruvate formate-lyase gene: upstream regulatory sequences and multiple promoters regulate anaerobic expression. J Bacteriol 171, 2485-2498.

Sawers, G. \& Suppmann, B. (1992). Anaerobic induction of pyruvate formate-lyase gene expression is mediated by the ArcA and FNR proteins. J Bacteriol 174, 3474-3478.

Sawers, R. G. \& Clark, D. P. (2004). Fermentative pyruvate and acetyl CoA metabolism. In EcoSal - Escherichia coli and Salmonella Cellular and Molecular Biology, chapter 3.5.3; www.ecosal.org (posted July 2004). Editor-in-Chief, R. Curtiss, III. Washington, DC: American Society for Microbiology.

Suppmann, B. \& Sawers, G. (1994). Isolation and characterisation of hypophosphite-resistant mutants of Escherichia coli: identification of the FocA protein, encoded by the $p f l$ operon, as a putative formate transporter. Mol Microbiol 11, 965-982.

Tock, M. R., Walsh, A. P., Carroll, G. \& McDowall, K. J. (2000). The CafA protein required for the 5 -maturation of $16 \mathrm{~S}$ rRNA is a 5 '-end-dependent ribonuclease that has context-dependent broad sequence specificity. J Biol Chem 275, 8726-8732.

Towbin, H., Staehelin, T. \& Gordon, J. (1979). Electrophoretic transfer of proteins from polyacrylamide gels to nitrocellulose sheets: procedure and some applications. Proc Natl Acad Sci U S A 76, 4350-4354.

Umitsuki, G., Wachi, M., Takada, A., Hikichi, T. \& Nagai, K. (2001). Involvement of RNase $\mathrm{G}$ in in vivo mRNA metabolism in Escherichia coli. Genes Cells 6, 403-410.

Wachi, M., Umitsuki, G. \& Nagai, K. (1997). Functional relationship between Escherichia coli RNase E and the CafA protein. Mol Gen Genet 253, 515-519.

Wagner, A. F. V., Frey, M., Neugebauer, F. A., Schäfer, W. \& Knappe, J. (1992). The free-radical in pyruvate formate-lyase is located on glycine-734. Proc Natl Acad Sci U S A 89, 996-1000. 